\title{
Treatment of Acute Kidney Injury Using a Dual Enzyme Embedded Zeolitic Imidazolate Frameworks Cascade That Catalyzes In Vivo Reactive Oxygen Species Scavenging
}

\section{OPEN ACCESS}

Edited by:

Jianxun Ding,

Changchun Institute of Applied

Chemistry (CAS), China

Reviewed by:

Xu Zhen,

Nanjing University, China Hani Nasser Abdelhamid,

Assiut University, Egypt

Yu Tao,

Sun Yat-sen University, China

*Correspondence: Tingting Huang

huangtt18@mails.jlu.edu.cn Jiancheng Guo gjc@zzu.edu.cn Wenjun Shang

fccshangwj@zzu.edu.cn

tThese authors have contributed equally to this work

Specialty section: This article was submitted to Biomaterials,

a section of the journal Frontiers in Bioengineering and Biotechnology

Received: 23 October 2021 Accepted: 01 December 2021 Published: 04 January 2022

Citation:

Hou X, Shi J, Zhang J, Wang Z,

Zhang S, Li R, Jiang W, Huang T, Guo J and Shang W (2022) Treatment of Acute Kidney Injury Using a Dual

Enzyme Embedded Zeolitic Imidazolate Frameworks Cascade That Catalyzes In Vivo Reactive Oxygen

Species Scavenging.

Front. Bioeng. Biotechnol. 9:800428.

doi: 10.3389/fbioe.2021.800428
Xinyue Hou ${ }^{1,2 \dagger}$, Jianxiang Shi ${ }^{2 \dagger}$, Jie Zhang ${ }^{1}$, Zhigang Wang ${ }^{1}$, Sen $\mathrm{Zhang}^{2}$, Ruifeng $\mathrm{Li}^{2}$, Wei Jiang ${ }^{2}$, Tingting Huang ${ }^{3 *}$, Jiancheng Guo ${ }^{2 *}$ and Wenjun Shang ${ }^{1 *}$

${ }^{1}$ Department of Kidney Transplantation, The First Affiliated Hospital of Zhengzhou University, Academy of Medical Sciences, Zhengzhou University, Zhengzhou, China, ${ }^{2}$ Department of Molecular Pathology, Application Center for Precision Medicine, The Second Affiliated Hospital of Zhengzhou University, Academy of Medical Sciences, Zhengzhou University, Zhengzhou, China, ${ }^{3}$ College of Chemistry, Jilin University, Changchun, China

Significant advances have been made in recent years for the utilization of natural enzymes with antioxidant properties to treat acute kidney injury (AKI). However, these enzymes have been of limited clinical utility because of their limited cellular uptake, poor pharmacokinetic properties, and suboptimal stability. We employed a novel biomimetic mineralization approach to encapsulate catalase (CAT) and superoxide dismutase (SOD) in a zeolitic imidazolate framework-8 (ZIF-8). Next, this SOD@CAT@ZIF-8 complex was anchored with $\mathrm{MPEG}_{2000}-\mathrm{COOH}$ to yield an $\mathrm{MPEG}_{2000}-\mathrm{SOD} @ \mathrm{CAT} @ Z I F-8$ (PSCZ) composite. The composite was then used as a stable tool with antioxidant properties for the integrated cascade-based treatment of $\mathrm{AKI}$, remarkably improved intracellular enzyme delivery. This dual-enzyme-embedded metal-organic framework could effectively scavenge reactive oxygen species. In conclusion, the ZIF-8-based "armor plating" represents an effective means of shielding enzymes with improved therapeutic utility to guide the precision medicine-based treatment of AKI.

Keywords: metal-organic frameworks, biomimetic mineralization, dual-enzyme cascade, ROS scavenging, acute kidney injury

\section{BACKGROUND}

Acute kidney injury (AKI) is a severe condition wherein glomerular filtration is impaired, leading to the accumulation of nitrogenous waste in the systemic circulation (Bellomo et al., 2012). Over time, AKI can progress to end-stage renal disease (ESRD), which affects up to $20 \%$ of hospitalized patients and results in high morbidity and mortality rates (Susantitaphong et al., 2013). Clinicians often encounter two forms of AKI, such as rhabdomyolysis-induced AKI (RM-AKI) or cisplatin-induced AKI (CP- AKI), although other instances exist (Arany and Safirstein, 2003). RM- AKI often occurs after the breakdown and necrosis of skeletal muscle releases pernicious proteins, largely myoglobin, and electrolytes into circulation. CP-AKI frequently arises from the nephrotoxicity associated with the use of cisplatin in the cancer patients, which occurs in about $1 / 3$ of all patients (Pabla and Dong, 2008). Oxidative stress leading to acute tubular necrosis from reactive oxygen species (ROS) has been implicated in the pathogenesis of both conditions, as in many cases of AKI. 
Reactive oxygen species (ROS), such as singlet oxygen $\left({ }^{1} \mathrm{O}_{2}\right)$, superoxide anions $\left(\mathrm{O}_{2}\right)$, hydroxyl radicals $\left({ }^{\circ} \mathrm{OH}\right)$, and hydrogen peroxide $\left(\mathrm{H}_{2} \mathrm{O}_{2}\right)$, are the metabolites of normal cells in living organisms (Yasui and Sakurai, 2000; Ighodaro and Akinloye, 2018). Healthy kidney tissues can tolerate limited ROS levels with no adverse effects. However, excessive ROS can trigger a series of destructive processes in normal cells, including cell death and inflammation (Nath and Norby, 2000; Molitoris et al., 2007). Patients with AKI have excessive oxidative stress due to increased oxide activity and decreased antioxidant activity in their serum, leading to renal dysfunction. Unfortunately, no effective clinical treatments for AKI have been developed to date, and most of the patients have to seek supportive treatment via hemodialysis, peritoneal dialysis, and kidney transplantation. So how to prevent and treat AKI plays a pivotal role in clinical settings (Paller et al., 1984; Sureshbabu et al., 2015; Du et al., 2017).

Eliminating excess ROS can protect tissues from oxidative stress, and effectively scavenging excess ROS can, therefore, have a significant therapeutic effect on AKI (Dennis and Witting, 2017).

A range of antioxidant enzymes, including superoxide dismutase (SOD), catalase (CAT), and reduced glutathione (GSH), can function as ROS scavengers to mitigate oxidative stress within a given cellular environment (Savelieff et al., 2019; Wu et al., 2019). Given that they are enzymes rather than small molecule therapeutics, these enzymes can catalyze the sustained conversion of toxic ROS species into less damaging byproducts, with SOD facilitating the conversion of ${ }^{\bullet} \mathrm{O}_{2}{ }^{-}$into $\mathrm{H}_{2} \mathrm{O}$ and then CAT converting $\mathrm{H}_{2} \mathrm{O}_{2}$ into water and $\mathrm{O}_{2}$ (Ferreira et al., 2018). While they can function in concert, CAT is primarily within peroxisomes, whereas SOD is typically in the cytosol or mitochondria, so additional transportation is necessary to facilitate $\mathrm{H}_{2} \mathrm{O}_{2}$ degradation (Sun et al., 2018; Wang et al., 2019). These endogenous enzyme systems show promise for in vivo therapeutic ROS scavenging and associated AKI treatment as adequate and well-controlled antioxidants. Such enzymatic cascade reactions have advantages over conventional multistage reactions, reducing barriers to diffusion while increasing local intermediate concentrations to enhance overall reaction efficiency at a molecular level. These good therapeutic properties have led many research groups to design enzymatic scaffold systems to mimic these efficient cascade systems in clinical settings. The in vivo application of these enzyme-based platforms would represent a key breakthrough with the potential to improve patient outcomes. However, several significant barriers currently limit the in vivo deployment of these enzyme-based systems, including the fact that they can readily undergo enzymatic degradation, are prone to enzyme aggregate formation, lose catalytic activity throughout prolonged storage, and exhibit poor uptake by target cells owing to their negatively charged surfaces. Embedding or encapsulating these enzymes in a solid carrier framework thus offers an opportunity to overcome these delivery and stability limitations.

Metal-organic frameworks (MOFs) are novel biocompatible materials that exhibit tunable porosity and a high surface area, making them ideal for the packaging of candidate therapeutic biomolecules (Simon-Yarza et al., 2017; Lu et al., 2018; Suresh and Matzger, 2019; Rosenkrans et al., 2020). These merits endow
MOFs with considerable potential to host a wide variety of guests from gas molecules, biomicromolecules to nanoparticles for advanced applications. For example, enzymes are a kind of bionanopaticles and hosting enzymes using MOFs is an emerging immobilization biotechnology that circumvents the low loading efficiency, incompact confinement, and restricted pore size of the supports drawbacks caused by conventional porous solids. Traditionally, four primary strategies have been used for such packaging: grafting, infiltration, physical absorption, and encapsulation (Feng et al., 2015; Shieh et al., 2015; Jiang et al., 2017; Chen et al., 2021). However, these approaches do not allow for precise control of the size of the generated biomacromolecular-MOF composites and are often highly inefficient. In the past few decades, anchoring enzymes to the outer surface of MOF particles by chemical grafting or physical adsorption has been the primary method for entrapment of the enzymes (Rosenkrans et al., 2020; Tripathy et al., 2021). It relied on the interaction between the organic ligand of MOFs and the surface components of enzymes (Liu et al., 2021; Velásquez-Hernández et al., 2021; Xu et al., 2021). Although this method provides a certain protection against enzyme denaturation, most enzymes are still directly exposed, so immobilized enzymes are still susceptible to changes in the external environment (Liang et al., 2021; Velásquez-Hernández et al., 2021). Using the biomimetic mineralization strategy, encapsulating the enzyme within MOFs cavities (enzymes@ MOFs) offered a aolution to the above problems (Liang et al., 2015; Rosenkrans et al., 2020). Leveraging the fact that biomacromolecule exhibit an affinity for metal ions to conduct rapid macromolecule embedding a MOF through the stimulation of zeolitic imidazolate framework-8 (ZIF-8) growth. This approach can shield the enclosed macromolecules within a porous ZIF-8 exoskeleton capable of protecting against denaturation while permitting selective transportation through this porous network structure. ZIF-8 exoskeletal constructs are highly stable and biocompatible under physiological conditions, while the entrapped enzymes were feasible to be released through simple modification of $\mathrm{pH}$ owing to the acid-sensitive nature of ZIF-8. The occluded enzymes could well maintain their activity. Most studies of such MOF bio-composites to date have explored their use as biosensors or catalytic agents in aqueous or organic solutions (Sun et al., 2012; Wang et al., 2017; Huang et al., 2020). Using these MOFs to deliver proteins or nucleic acids is, in contrast, a relatively novel approach (Zhuang et al., 2020a; Zhuang et al., 2020b). To our knowledge, no prior studies described the biomimetic mineralization-based encapsulation of dual-enzyme within MOF crystals to enhance ROS scavenging. We hypothesized that a ZIF-8 exoskeleton would protect SOD and CAT (SOD@CAT@ZIF-8), improve their stability, and overcome the current barriers to alleviate ROS-mediated renal damage.

In the present study, we aim to develop a MOF encapsulating key antioxidant enzymes using a nanoscale ZIF-8 exoskeleton scaffold containing SOD and CAT (SOD@CAT@ZIF-8). This platform was further functionalized by anchoring $\mathrm{MPEG}_{2000^{-}}$ $\mathrm{COOH}$ to the surface of the MOF complex, yielding more stable and biocompatible composites (Scheme 1). Co-delivery of SOD and CAT enzymes can also be achieved using technology by 


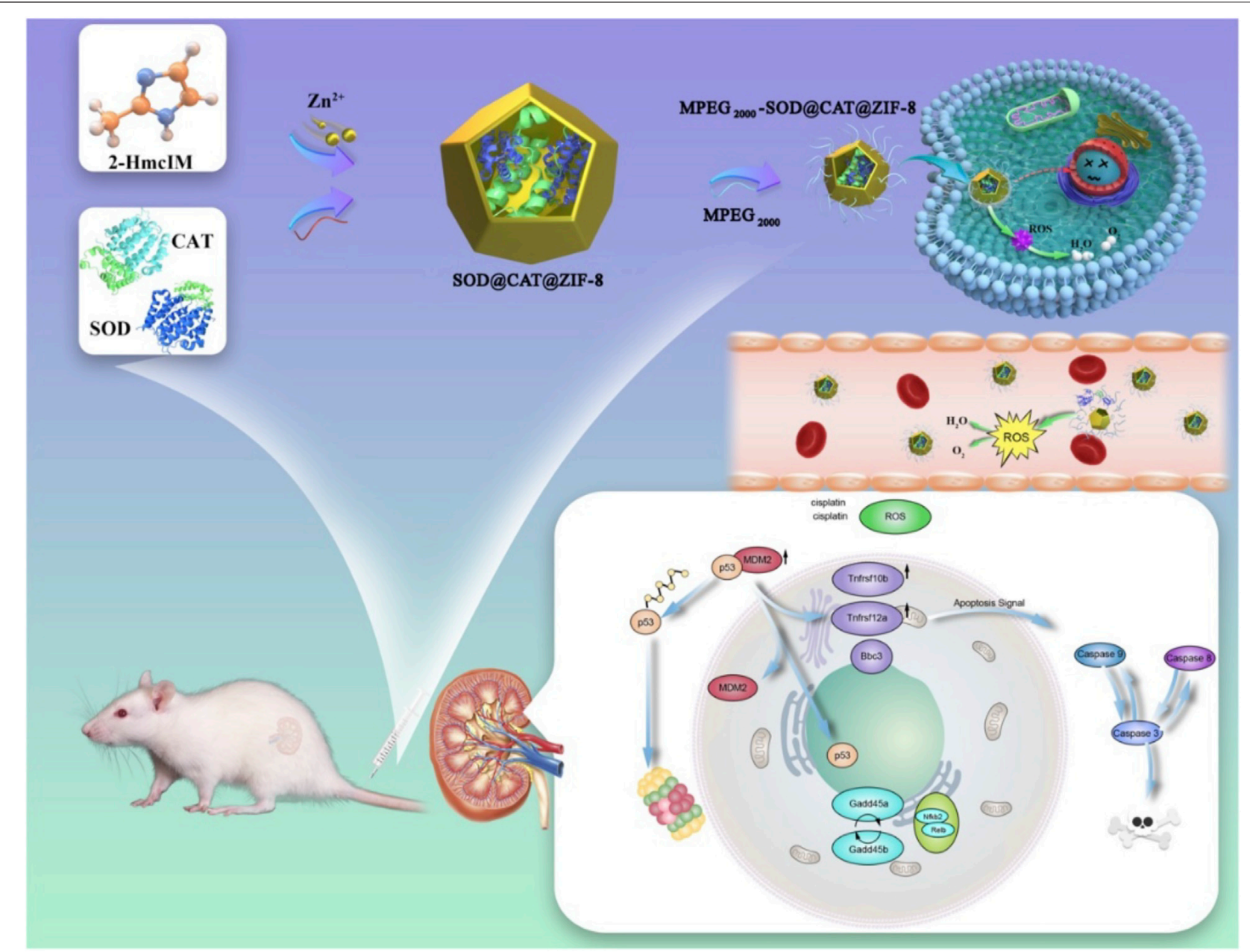

SCHEME 1 | Schematic overview of PSCZ and their ROS scavenging application through a combination of SOD and CAT.

linking the enzymes into enzyme complexes, followed by in situ free-radical polymerization. Different from these previous arts, this work uses a zeolitic imidazolate framework- 8 to form enzyme-polymer nano complexes followed by an MPEG to encapsulate the nano complexes under mild conditions, resulting in the formation of multiple-enzyme nanoparticles. Relative to free CAT and SOD, this integrated $\mathrm{MPEG}_{2000^{-}}$ SOD@CAT@ZIF-8 (PSCZ) platform exhibited, more significant SOD and CAT enzymatic efficiency in vitro and was found to enhance ROS scavenging in vivo, protecting mice against AKIassociated oxidative renal tissue damage. Together, our data show MOF platforms incorporating integrated enzyme cascades and offer great promise for the in vivo biomedical treatment of AKI.

\section{RESULTS AND DISCUSSION}

\subsection{Synthesis and Characterization of PSCZ}

As shown in Scheme 1, the native enzyme could be embedded in a porous nanomaterial ZIF-8, in which $\mathrm{Zn}(\mathrm{OAc})_{2}, 2-\mathrm{HMeIM}$, SOD, and CAT were incubated for $5 \mathrm{~min}$ at room temperature. The morphology of composite SOD@CAT@ZIF8 and PSCZ was characterized by SEM (Figure 1A). The SEM image showed that SOD@CAT@ZIF-8 and PSCZ both possessed uniform rhombic dodecahedral morphology similar to blank ZIF8. As shown in Figure 1B, the PXRD pattern of $\mathrm{MPEG}_{2000}-\mathrm{SOD} @$ ZIF-8 (PSZ), MPEG 2000 -CAT@ZIF-8 (PCZ), and PSCZ both exhibited peaks matching well with the simulated ZIF-8 suggesting that ZIF-8 frameworks were formed in the biomimetic mineralization, and the structural integrity of ZIF8 could be kept in the presence of SOD and CAT. FT-IR spectra of PSZ, PCZ, and PSCZ characteristic with $\mathrm{MPEG}_{2000}-\mathrm{COOH}$ peaks at $2,865-2,875 \mathrm{~cm}^{-1}$ that were respectively attributable to $-\mathrm{COOH}$ and $-\mathrm{OCH}_{3}$, indicating the successful conjunction of $\mathrm{MPEG}_{2000}$.

In order to prove that the SOD enzyme and CAT enzyme are encapsulated in the same crystal with ZIF-8, we used fluorescein isothiocyanate (FITC) to label SOD and Rhodamine 6G to label CAT. Detect the fluorescence spectra of the mixture (denoted as SOD+CAT) and PSCZ separately. As shown in Supplementary Figure S1, under an excitation wavelength of $450 \mathrm{~nm}$, both the samples show emission at $520 \mathrm{~nm}$ attributed to FITC, whereas 

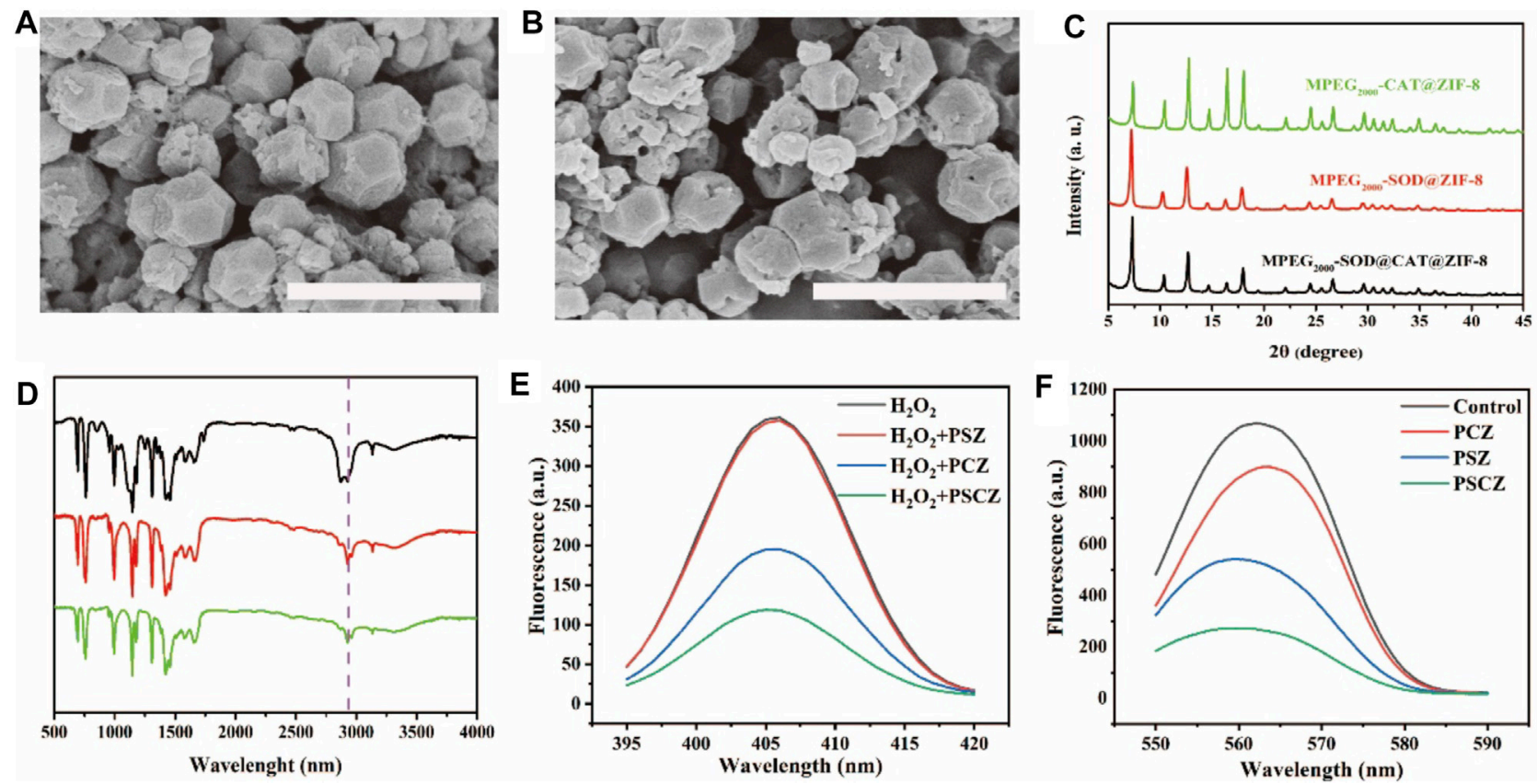

FIGURE 1 | (A,B) SEM images of SOD@CAT@ZIF-8 and PSCZ following rinsing using $\mathrm{dH}_{2} \mathrm{O}$; scale bar = $1 \mu \mathrm{m}$; (C) Powder X-ray diffraction (PXRD) patterns of PSZ, PCZ, and PSCZ; (D) Fourier transform infrared spectra (FT-IR) of PSZ (green), PCZ (red), and PSCZ (black); (E) CAT-like activity for PSZ, PCZ, and PSCZ; (F) Fluorescence spectra for SOD-like activity of PSZ, PCZ, and PSCZ with concentration $(100 \mu \mathrm{g} / \mathrm{ml})$.

PSCZ exhibits an extra emission at $580 \mathrm{~nm}$ attributed to Rhodamine 6G, showing that the SOD enzyme and CAT enzyme are encapsulated in the same crystal of ZIF-8.

We further investigated the capacity to scavenge various free radicals of PSCZ, PSZ, and PCZ. Among biologically relevant ROS, $\mathrm{H}_{2} \mathrm{O}_{2}$ is of greatest importance because of its membrane permeability, longer half-life than $\mathrm{O}^{2-}$ and ${ }^{\circ} \mathrm{OH}$, and consequently highest intracellular concentration. CAT can catalyze the decomposition of $\mathrm{H}_{2} \mathrm{O}_{2}$ into $\mathrm{H}_{2} \mathrm{O}$ and $\mathrm{O}_{2}$, protecting cells and tissues from oxidative damage. The CATlike activity of PCZ, PSZ free enzyme, and PSC cascade enzyme was investigated by recording the changes in absorbance of $\mathrm{H}_{2} \mathrm{O}_{2}$. We add xanthine oxidase, horseradish peroxidase, water-soluble tetrazolium salt (WST-1), and amplex red are added to enzyme solutions containing PSZ, PCZ, or PSCZ. Xanthine oxidase catalyzes the oxidation of WST-1, generating superoxide that is converted to $\mathrm{H}_{2} \mathrm{O}_{2}$ through the mediation of SOD. Meanwhile, mediated by horseradish peroxidase and $\mathrm{H}_{2} \mathrm{O}_{2}$, amplex red is converted to resorufin, a fluorescence probe for the $\mathrm{H}_{2} \mathrm{O}_{2}$ concentration of the solution. As shown in Figure 1E, the lower the absorbance, the stronger the scavenging ability of $\mathrm{H}_{2} \mathrm{O}_{2}$. The CAT-like activity of the PSCZ showed the best results within absorbance at $405 \mathrm{~nm}$. SOD-like enzymes can treat a variety of oxidative stress-related diseases. The SODlike ability of the PSCZ to eliminate $\mathrm{O}_{2}{ }^{--}$was measured using formazan, produced by the reaction of $\mathrm{O}_{2}{ }^{--}$and nitrogen blue tetrazole. The specific absorption of formazan was measured at $560 \mathrm{~nm}$. The absorbance of formazan by PCZ, PSZ and PSCZ was detected at the same concentration, and the absorbance of PSCZ was far lower than PCZ and PSZ. The results showed that PSCZ had superior SOD-like enzyme activity.

Natural enzymes often exhibit intrinsic shortcomings, such as low operational stability, temperature, and $\mathrm{pH}$ sensitivity. Hence, the thermal and $\mathrm{pH}$ stabilities of PSCZ were investigated and compared with those of the natural enzyme SOD and CAT. The results (Supplementary Figures S2, S3) suggested that the stabilities of PSCZ when exposed to $\mathrm{pH}$ and temperature variations were significantly greater than those of natural SOD and CAT. These results show the increase in enzyme activity after biomimetic mineralization.

\subsection{Assessment of the Ability of PSCZ to Scavenge Reactive Oxygen Species In Vitro}

The kidney is vulnerable to damage from ROS because it receives approximately $25 \%$ of the blood supply. The imbalance of ROS, referred to as oxidative stress, is detrimental to renal tubules and ultimately leads to AKI. Given the promising properties of PSCZ particles as stable, biocompatible antioxidant agents, we next assessed their ROS scavenging activity in vitro relative to that of free CAT and SOD. Given that AKI pathogenesis is associated with oxidative damage to the renal tubules, we used the HEK293 cell line model to assess the ability of PSCZ to protect against ROS-mediated injury (Pavlakou et al., 2017). $\mathrm{H}_{2} \mathrm{O}_{2}$ reacts to generate free radicals responsible for the cause of cell damage. Therefore, the ability of PSZ, PCZ, free SOD, free CAT, ZIF- 8 and PSCZ to reduce the levels of $\mathrm{H}_{2} \mathrm{O}_{2}$ and ROS production was investigated in HEK293 cells. Cell viability test reveals PSC, PCZ, 


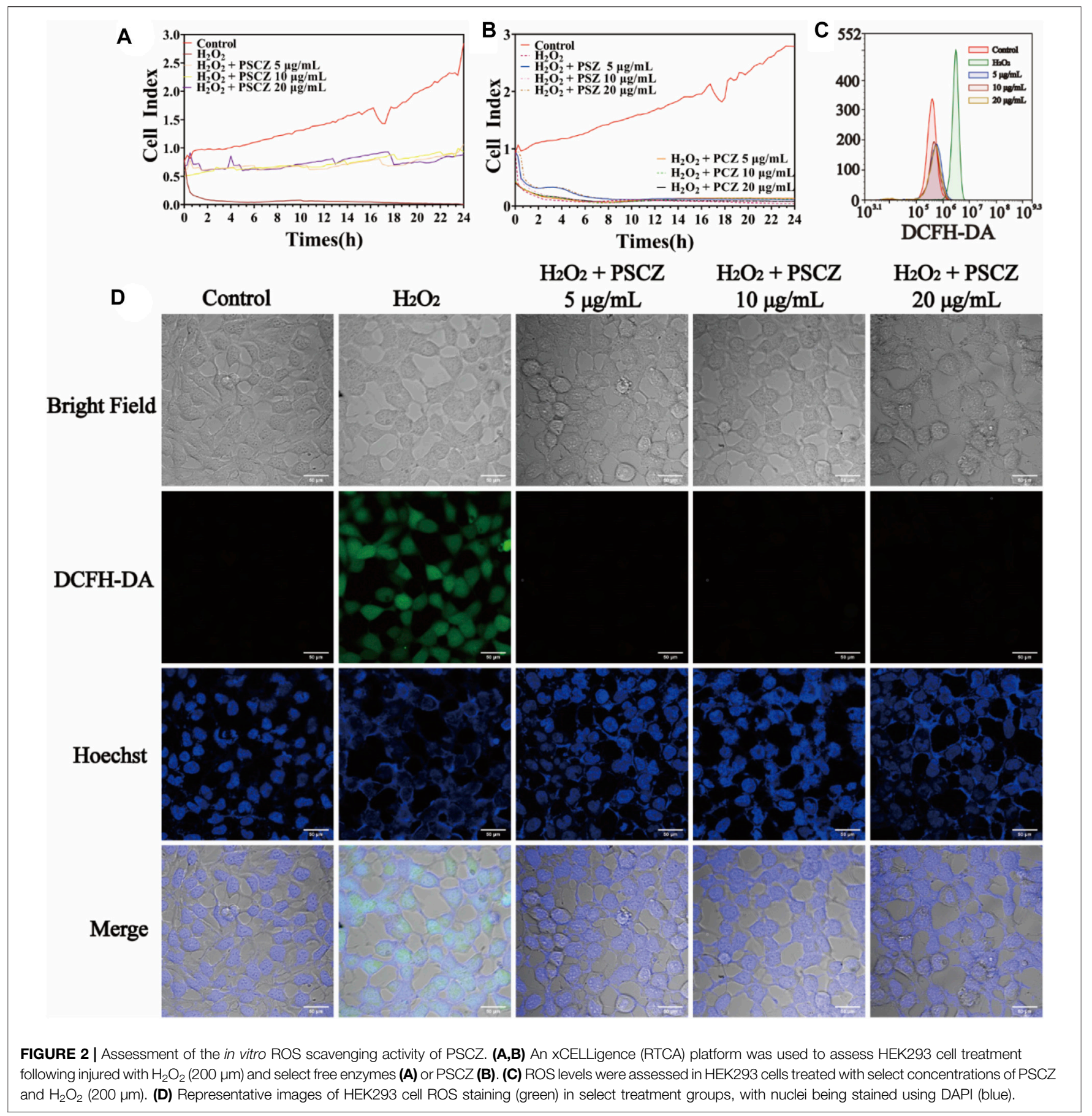

free SOD, free CAT, and ZIF-8 had no protective effect on cells under the stimulation of $\mathrm{H}_{2} \mathrm{O}_{2}(200 \mu \mathrm{m})$ injury, while PSCZ significantly improves cell viability (Supplementary Figure S4; Supporting Information). In order to further compare the cell protective effect of PSCZ, we additionally used an xCELLigence (RTCA) instrument to monitor the survival and growth of these cells in a quantitative, real-time, dynamic manner. This assay approach is superior to traditional endpoint-based viability assays that assess membrane permeability (Yah and Simate, 2020), enabling the assessment of cell survival in a label-free and automated manner throughout a given experimental period (Cetin and Topcul, 2019). RTCA approach was used to measure the survival and growth of cells over a $24 \mathrm{~h}$ period following injury with $\mathrm{H}_{2} \mathrm{O}_{2}$ and these different nanoenzymes preparations. PSCZ showed superior antioxidant capacity at $5 \mu \mathrm{g} /$ $\mathrm{ml}$ than PSZ and PCZ even at a higher $(20 \mu \mathrm{g} / \mathrm{ml})$ concentration (Figure 2A). We then injured HEK293 cells with $\mathrm{H}_{2} \mathrm{O}_{2}$ to induce oxidative stress to evaluate the ability of PSCZ to protect against oxidative damage (Figure 2B). While the viability of $\mathrm{H}_{2} \mathrm{O}_{2}$ treated cells was $\sim 50 \%$, PSCZ treatment was associated with a 


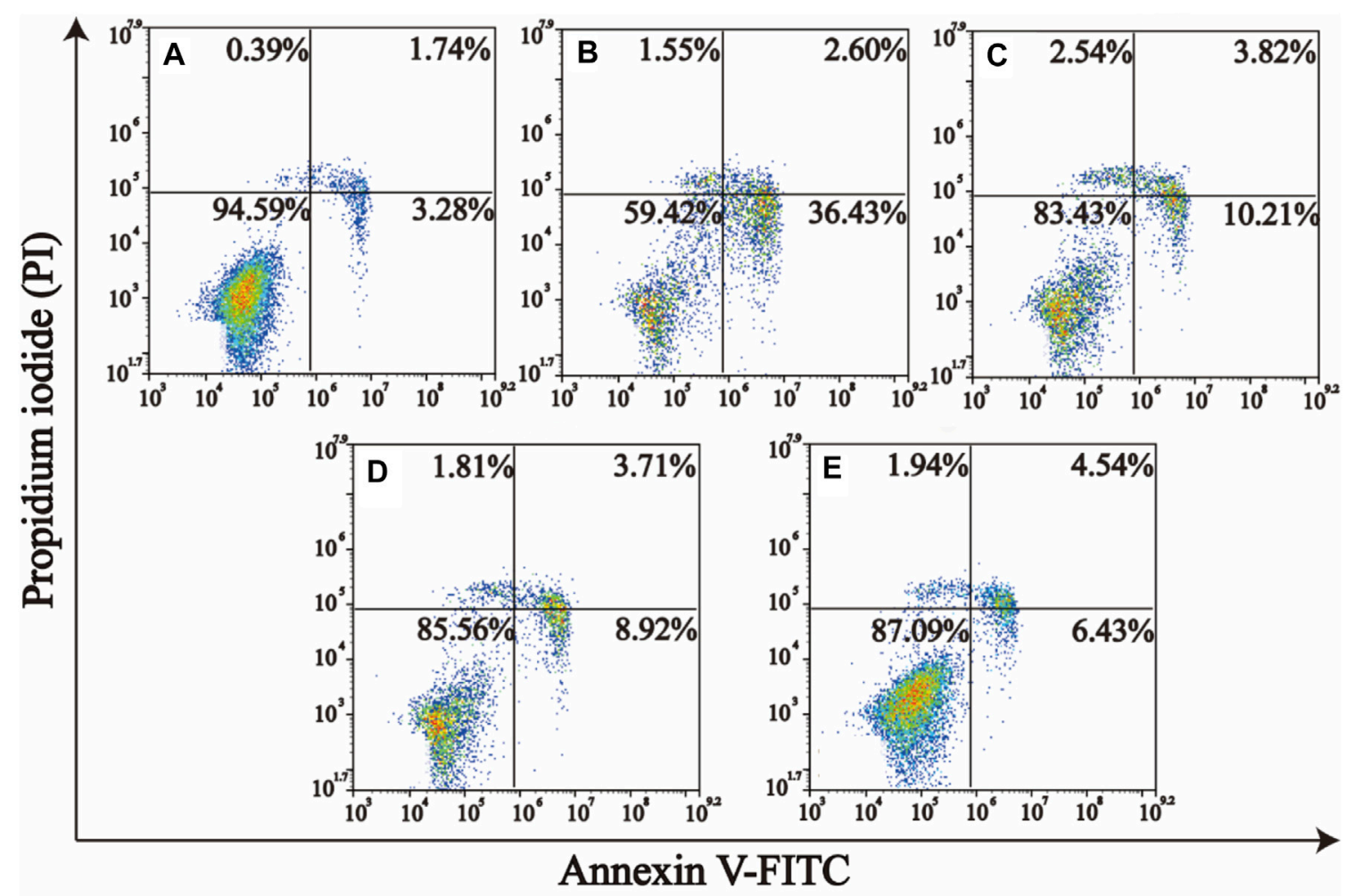

FIGURE 3 | PSCZ protects HEK293 cells against oxidative damage induced by $\mathrm{H}_{2} \mathrm{O}_{2}$. AnnexinV-fluorescein isothiocyanate (FITC)/PI dual-staining was used to assess the impact of various PSCZ concentrations on apoptotic cell death in $\mathrm{HEK} 293$ cells that had been treated for $24 \mathrm{~h}$ with $\mathrm{H}_{2} \mathrm{O}_{2}$ (200 $\mu \mathrm{m}$ ). (A) Control group; (B) $\mathrm{H}_{2} \mathrm{O}_{2}$ injury $(200 \mu \mathrm{m}) ;$ (C) $\mathrm{H}_{2} \mathrm{O}_{2}(200 \mu \mathrm{m})+\mathrm{PSCZ} 5 \mu \mathrm{g} / \mathrm{ml}$; (D) $\mathrm{H}_{2} \mathrm{O}_{2}(200 \mu \mathrm{m})+\mathrm{PSCZ} 10 \mu \mathrm{g} / \mathrm{ml} ;$ (E) $\mathrm{H}_{2} \mathrm{O}_{2}(200 \mu \mathrm{m})+\mathrm{PSCZ} 20 \mu \mathrm{g} / \mathrm{ml}$.

significant increase in these cells' survival because of the observed reductions in intracellular ROS production and the consequent preservation of mitochondrial functionality (Figure 2B).

We also used the DCFH-DA fluorescent probe to assess intracellular ROS levels to further confirm whether PSCZ could suppress $\mathrm{H}_{2} \mathrm{O}_{2}$-induced ROS generation in HEK293 cells. As shown in Figure 2D, it shows that $\mathrm{H}_{2} \mathrm{O}_{2}$ injury of HEK293 cells stimulates ROS production, while in $\mathrm{PSCZ} / \mathrm{H}_{2} \mathrm{O}_{2}-$ injured cells reduced ROS production was observed. These results show that, compared with other groups, PSCZ can effectively reduce the generation of free radicals mediated by $\mathrm{H}_{2} \mathrm{O}_{2}$. PSCZ is safe for in vitro and in vivo applications. This finding was further confirmed via flow cytometry (Figure 2C), revealing that PSCZ could reliably suppress $\mathrm{H}_{2} \mathrm{O}_{2}$-mediated free radical generation.

Mitochondrial oxidative phosphorylation is the primary mechanism whereby most cells produce ATP (Xu et al., 2019). Given that renal PTECs lack glycolytic activity and are under high metabolic energy demands, these cells exist under conditions of ischemia and hypoxia (Yu et al., 2018). This increases the susceptibility of these renal tubular epithelial cells to acute renal injury. Mitochondria are also central regulators of intracellular apoptotic signaling, with the loss of mitochondrial membrane potential $(\Delta \psi \mathrm{m})$ being an early apoptotic indicator (Sastre et al., 2000). Therefore, we assessed the impact of PSCZ on HEK293 cell $\Delta \psi \mathrm{m}$ using a JC-1 staining approach, with a higher red/green JC-1 ratio indicative of lower levels of mitochondrial dysfunction. We observed a significant decrease in the $\Delta \psi \mathrm{m}$ of these cells following $\mathrm{H}_{2} \mathrm{O}_{2}$ injury (Supplementary Figure S5), whereas PSCZ treatment yielded a JC-1 fluorescence ratio comparable to that in control cells, suggesting that PSCZ can preserve mitochondrial membrane integrity.

Excess ROS generation can initiate apoptotic signaling cascades within cells (Ratliff et al., 2016; Meng et al., 2018). Given the acute sensitivity of renal tubule cells to oxidative damage, we next assessed the ability of PSCZ to facilitate in vitro ROS scavenging activity in HEK293 cell model. When the death of these cells was assessed via flow cytometry following Annexin V/PI staining, we found that $\mathrm{H}_{2} \mathrm{O}_{2}$ injured $(200 \mu \mathrm{m})$ increased the frequency of apoptotic HEK293 cells to $36.43 \%$, relative to control cells (3.28\%) (Figure 3). In contrast, cells treated with PSCZ exhibited significantly lower rates of apoptotic death $(6.43 \%)$. These findings thus confirmed that PSCZ could protect against oxidative damage in HEK293 cells in vitro.

\subsection{PSCZ Effectively Facilitates In Vivo ROS Scavenging}

Given the promising in vitro results detailed above, we next explored the in vivo utility of our MOF antioxidant platform using a murine cisplatin-induced AKI (CP-AKI) model system 


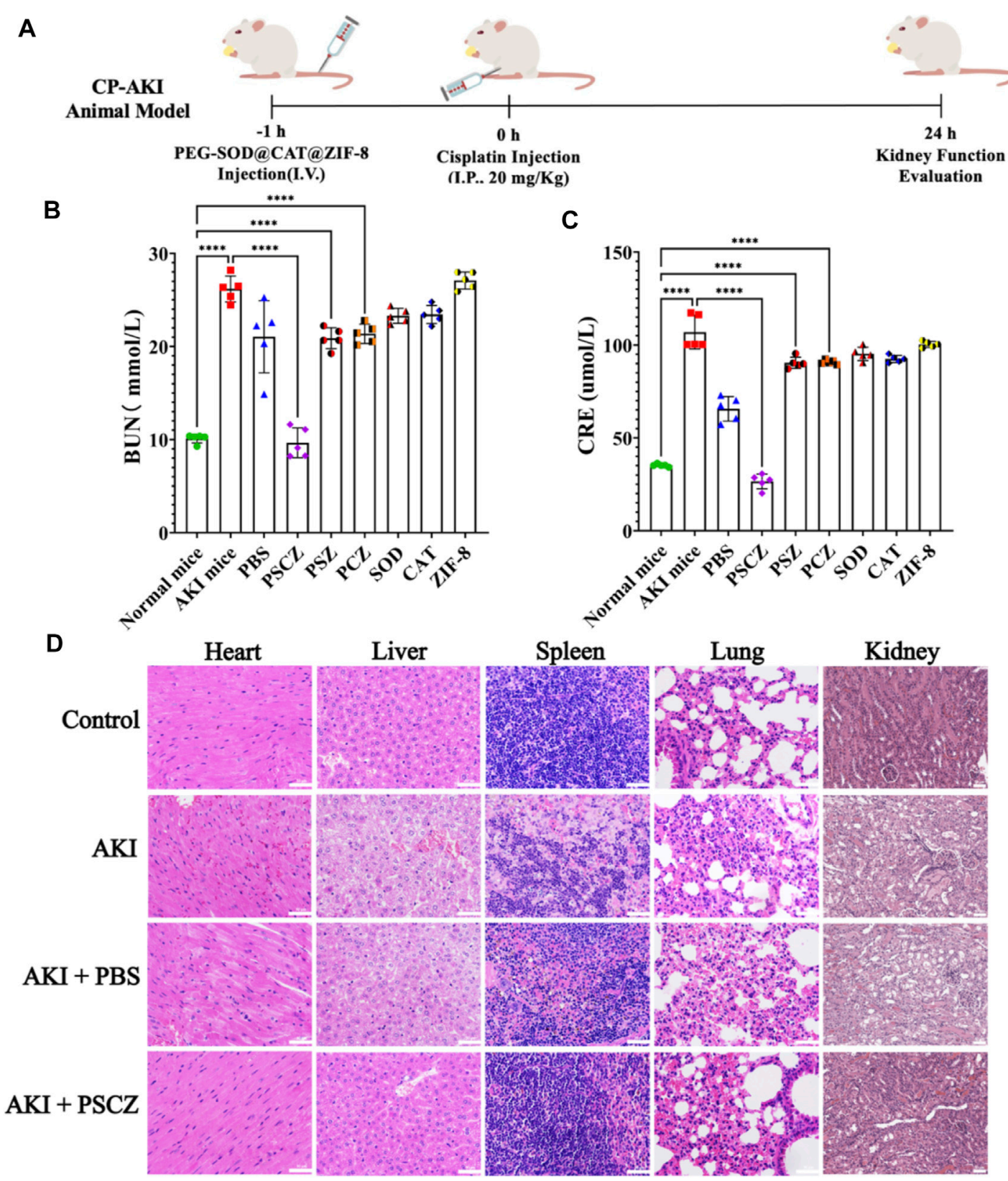

FIGURE 4 | Assessment of the biocompatibility of PSCZ in vivo. (A) CP-AKI modeling approach. (B) CRE levels in CP-AKI mice were measured at 24 h following select treatments. (C) BUN levels in CP-AKI mice were measured at $24 \mathrm{~h}$ following select treatments. (D) Tissues from select treatment groups were assessed following H\&E staining.

(Rewa and Bagshaw, 2014). Cisplatin (CDDP) is a widely used chemotherapeutic drug that accumulates within renal proximal tubule epithelial cells (PTECs), resulting in localized renal inflammation, damage, apoptotic death, and AKI that can manifest as CDDP-associated nephrotoxicity (Manohar and Leung, 2018). The pathogenesis of CP-AKI has been linked to ROS production and consequent acute tubular apoptosis (Ozkok and Edelstein, 2014). Thus, this CP-AKI model system was selected for further analyses of the relative safety and in vivo antioxidant activity of PSCZ.
The construction of the CP-AKI animal model is consistent with previous published articles (Ni et al., 2018; Sun et al., 2018; Rosenkrans et al., 2020; Yu et al., 2020). Briefly, CP-AKI mice were established as in prior reports by intraperitoneally injecting these animals with CDDP $(20 \mathrm{mg} / \mathrm{kg})$ and then measuring serum BUN and CRE levels after $24 \mathrm{~h}$ to gauge renal function (Bolisetty et al., 2016), given that these compounds accumulate in the blood in renal damage. To evaluate the effect of treatment in vivo, mice in corresponding treatment groups were intravenously treated with PSZ, PCZ, free SOD, free CAT, ZIF-8, and PSCZ $1 \mathrm{~h}$ before 

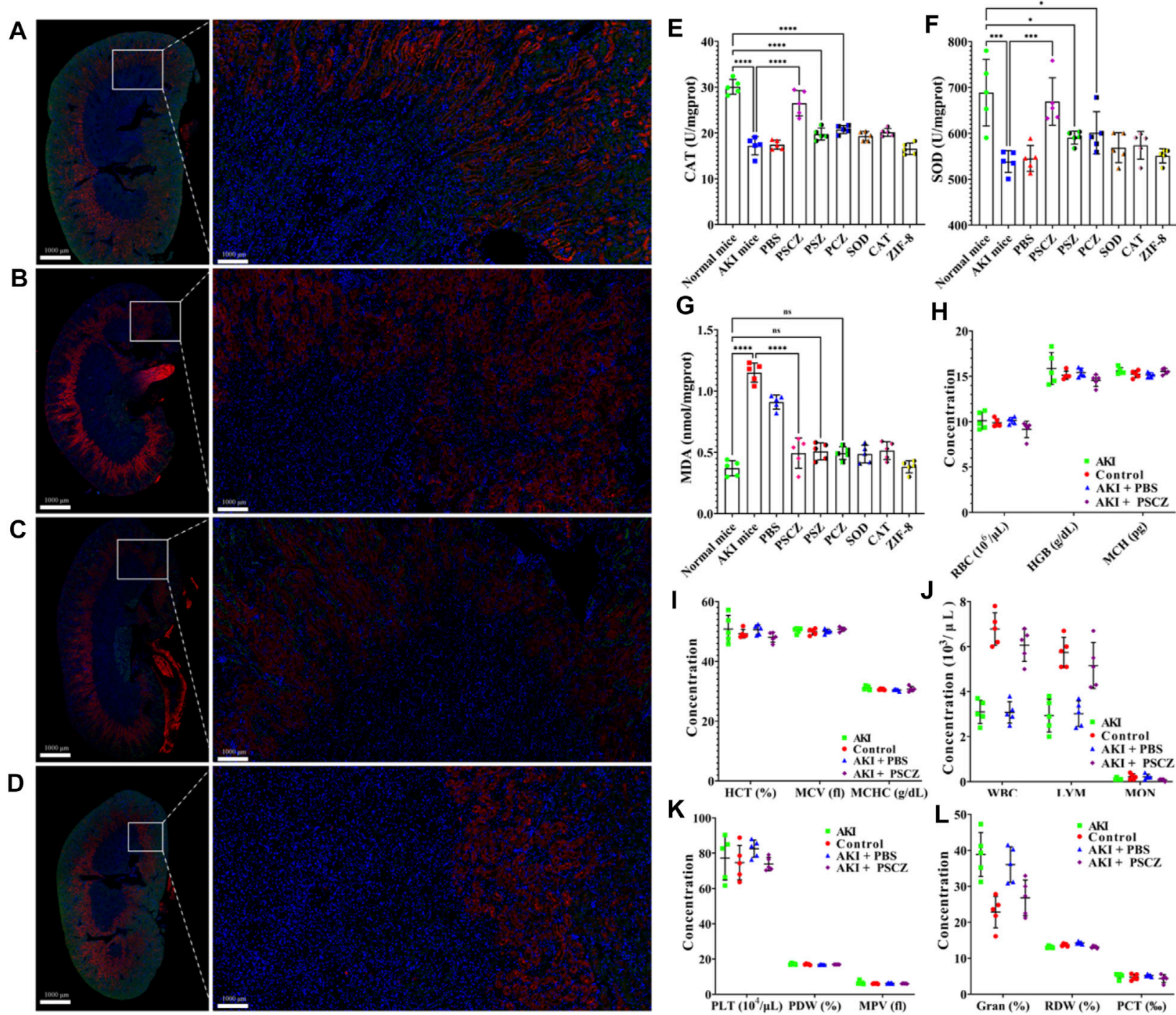

FIGURE 5 | Analysis of renal biomarker expression and ROS accumulation. (A-D) TNF- $\alpha$ and IL-1 $\beta$ levels in mice from the treatment groups were detected via immunofluorescent staining. (A) Control; (B) CP-AKI; (C) CP-AKI+PBS; (D) CP-AKI+PSCZ; (E) CAT levels in renal homogenates; (F) SOD levels in kidney homogenates; (G) MDA levels for mice in the groups; (H-L) The hematological parameters were assessed in murine treatment groups at $24 \mathrm{~h}$ post-PSCZ injection.

CDDP administration (Figure 4A), as Liu's research (Liu et al., 2020). Blood serum samples in each group were analyzed for CRE and BUN concentrations. CRE and BUN are nitrogenous waste products and accumulate in the blood when kidney function has been impaired. As a result, CRE and BUN are widely used in the clinic to assess kidney function. In CP-AKI mice, CRE and BUN levels were significantly higher in CP-AKI mice than in healthy controls (Figures 4B,C), consistent with the successful induction of CDDP-mediated kidney damage in these animals. Saline infusions are commonly administered to patients before and during CDDP treatment (Bolisetty et al., 2016) infusions exhibit poorly understood renoprotective activity against CDDP-induced nephrotoxicity. However, CP-AKI will manifest in roughly $30 \%$ of treated patients (Sharp et al., 2016). Our mouse model system observed comparable findings, as only slight reductions in CRE and BUN levels were observed in CP-AKI mice treated with PBS, with these levels being significantly higher than those in control animals. In contrast, the treatment of CP-AKI mice with PSCZ resulted in significant reductions in CRE and BUN levels to concentrations comparable to those in healthy control mice, however, free SOD, free CAT, PSZ, and PCZ mice in other groups had no significant therapeutic effect. These data underscored the therapeutic utility of PSCZ as a safe and effective tool for treating CP-AKI in mice.

We additionally isolated renal tissue sections from these treated mice and stained them with $H \& E$ stain to assess treatment-related changes in tissue pathology. Renal casts can arise due to denatured protein precipitation in kidney tubules and are often considered being an indicator of kidney disease. Similarly, inflammatory cell infiltration and the formation of 


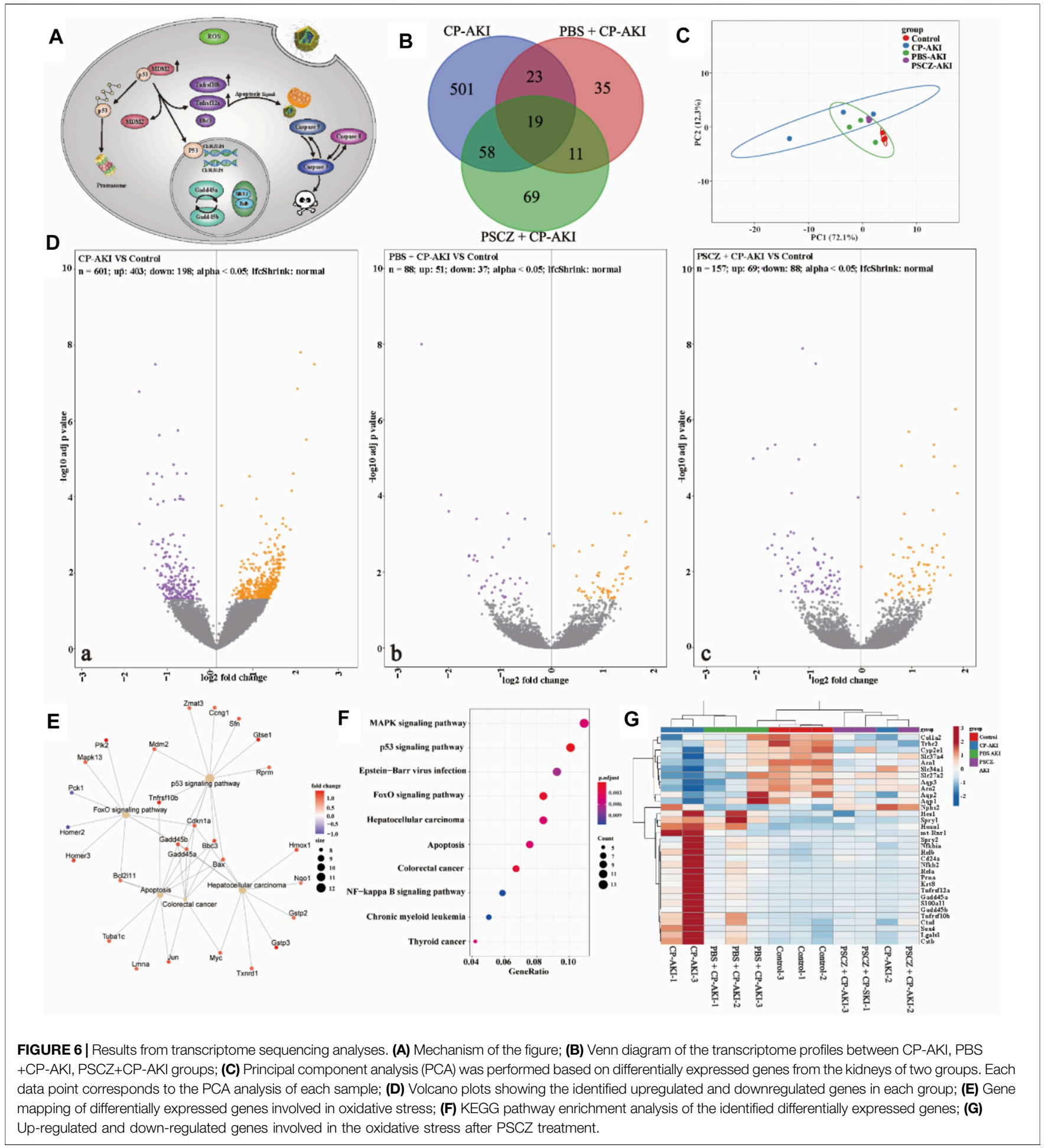

vacuoles within renal tubules can be visualized to detect and gauge the severity of the renal inflammatory injury. While many casts were clear in the renal tissue samples from CP-AKI model mice (Figure 4D; Supplementary Figure S8), relatively few were detectable in AKI model animals that had been administered PSCZ, consistent with the ability of these nanoparticles to preserve the integrity of renal tissues better. The H\&E staining of other tissues shows that PSCZ may have a therapeutic effect on inflammation of these tissues caused by cisplatin, but it is not within the scope of this study (Supplementary Figure S8).

To further assess the ability of PSCZ to suppress renal ROS generation, we next used the fluorescent ROS probe DCFH-DA to 
stain kidney tissue sections from CP-AKI mice in our different treatment groups and then imaged these tissues via laser scanning confocal microscopy. This approach revealed that PSCZ administration significantly inhibited renal ROS accumulation in our AKI model mice (Supplementary Figures S6, S7).

Tumor necrosis factor-alpha (TNF- $\alpha$ ) and interleukin-1 $\beta$ (IL$1 \beta)$ are key inflammatory cytokines and drivers of apoptotic cell death in cisplatin-induced renal injury (Martindale and Holbrook, 2002; Schieber and Chandel, 2014). Excess ROS production by cells under inflammatory conditions can induce additional proinflammatory cytokine production and promote further immune cell infiltration and renal damage. IL-1 $\beta$ can further amplify these inflammatory processes through feedback mechanisms (Ozkok and Edelstein, 2014). Renal tissue samples from treated mice were collected and analyzed to reveal the underlying mechanism of PSCZ. Immunofluorescence results showed that IL- $1 \beta$ and TNF- $\alpha$ levels were comparable in the PSCZ-treated and control groups, suggesting these nanoparticles did not induce direct inflammation in mice at the used concentrations and can reduce inflammation-induced kidney damage (Figures 5A-D). Notably, in AKI model mice, PSCZ administration suppressed TNF- $\alpha$ and IL- $1 \beta$ expression relative to untreated model controls. We additionally assessed SOD and CAT levels in renal homogenates from these mice (Figures 5E,F), to confirm that these antioxidant enzymes were successfully delivered to damaged kidney tissue (Hao et al., 2019). Excessive ROS levels can cause SOD and CAT depletion, as was clear in CP-AKI and PBS-treated mice. In contrast, PSCZ resulted in renal SOD and CAT levels similar to those in healthy control animals. It was confirmed that PSCZ was successfully delivered to the injured renal tissue and alleviated renal inflammatory injury.

To further test the therapeutic utility of PSCZ, we next measured malondialdehyde (MDA) levels and blood biochemical parameters to evaluate renal excretory function. MDA analysis results revealed that both CP-AKI mice and PBS-treated AKI mice exhibited elevated MDA levels consistent with renal failure, while PSCZ administration lowered these levels consistent with the alleviation of AKIrelated renal damage. Similar to other results, treated with PSZ, PCZ, free SOD, free CAT or ZIF-8 did not reduce MDA levels (Figure 5G).

Analyses of blood biochemical parameters from these mice showed that PSCZ treatment was not associated with obvious renal toxicity (Figures $\mathbf{5 H}-\mathbf{L}$ ), and hematological parameters in PSCZ-treated animals were comparable to those in healthy controls. These findings suggested that our PSCZ particles were highly biocompatible and useful as a tool for AKI treatment. For biocompatibility and biodistribution, we did not perform further repeated verification, because $\mathrm{He}$ et al. have conducted related research (Rosenkrans et al., 2020). However, it is essential to note that we only tested for acute toxicity associated with this therapeutic platform, and further research will be necessary to establish whether PSCZ is retained in the kidney beyond the $24 \mathrm{~h}$ time point, whether it is eliminated via renal metabolic processing, and whether it induces any longterm toxicity in vivo.

\subsection{RNAs-seq Analysis Confirmed the Successful Construction of the Acute Kidney Injury Model and PSCZ Showed a Superior Protective Effect in the Acute Kidney Injury Mouse Model}

In the current study, we set four different groups: control group with no treatment (brief as the control group), cisplatin-induced AKI group (brief as CP-AKI group), AKI group pre-treated with PSCZ (brief as the PSCZ+AKIgroup), and AKI group pre-treated with PBS (brief as the PBS+CP-AKI group). More detailed information is shown in the M\&M section.

An average of 63.34 (range from 58.51 to 66.33) million raw reads were obtained for all samples. After removing low-quality reads, an average of 60.52 (range from 56.05 to 63.16) million clean reads were retained for further analysis. The raw data were uploaded to National Omics Data Encyclopedia (NODE) database (https://www.biosino.org/node) with the following accession number: OEP000285.

To further elucidate the underlining therapeutic mechanisms, CP-AKI was chosen as the representative disease model for further transcriptome analysis. DESeq2 identified 601 DEGs, 157 DEGs, and 88 DEGs for the CP$\mathrm{AKI}$ group, $\mathrm{CP}-\mathrm{AKI}+\mathrm{PSCZ}$ group, and $\mathrm{CP}-\mathrm{AKI}+\mathrm{PBS}$ group compared with the control group, respectively (Figure 6D). An unguided principal component analysis (PCA) of the data revealed different transcriptome profiles between PSCZ and PBS-treated AKI mice kidneys (Figure 6C). The Venn diagram in Figure 6B showed many differentially expressed genes were produced in the CP-AKI group compared with the control group, and the differentially expressed genes were decreased in the PSCZ treatment group.

We also investigated the impact of PSCZ on gene expression related to growth arrest and DNA damage response, apoptosis, oxidation-reduction, etc. (Figure 6G). In brief, the PSCZ treatment could reverse the impact of cisplatin-induced AKI in the mouse model.

DEGs in the CP-AKI group were enriched in the p53 signaling pathway, FoxO signaling pathway, apoptosisrelated pathways, etc (Figures 6E,F; Supplementary Figure S9). This is consistent with previous research. Cisplatin can interfere with DNA replication and DNA repair mechanisms, cause DNA damage, and induce apoptosis in cells (Dasari and Bernard Tchounwou, 2014). Cisplatin can also increase cellular ROS and induce endoplasmic reticulum (ER) stress, contributing to cisplatin toxicity. Cell death and ER stress are characteristic of cisplatin-induced AKI (Sharp et al., 2016; Sharp and Siskind, 2017). P53 signaling is an early stage response to cisplatin toxicity in renal cells. Cisplatin caused renal cells to initiate the $\mathrm{p} 53$ dependent DNA repair pathway. If ROS continues to be overproduced in renal cells, the intrinsic apoptotic pathway will be activated. In the early stage, when cisplatin interferes with DNA replication and DNA repair mechanism, nano inhibits the excessive production of ROS caused by cisplatin, promotes the initiation of DNA repair pathway, and alleviates renal cell damage (Figure 6A; Supplementary Figure S10). 
In the current study, the kidney proximal nephron tubule segment injury marker Havcr1 (also known as Kim1) slightly changed $\left(\log _{2} \mathrm{FC}=0.42\right.$, adj. $\left.P=0.437\right) \mathrm{CP}-\mathrm{AKI}$ group compared with the control group. This is probably due to the kidneys were harvested in the very early stage of AKI. This is consistent with a previous study that cisplatin-induced AKI mainly caused proximal nephron tubule segment injury in the very early stage (Bolisetty et al., 2016). Bax, Bakl are important signals in the apoptosis cascade and normally act on the mitochondrial membrane to promote permeabilization and release of cytochrome $\mathrm{C}$ and ROS. In CP-AKI group, Bax and Bak1 significantly increased $\left(\log _{2} \mathrm{FC}=1.16\right.$, adj. $P=0.024 ; \log _{2} \mathrm{FC}=$ 0.99 , adj. $P=0.048$, respectively). This means that ROS level increased by cisplatin. Aco1 and Cyp2e1, which are important oxidation-reduction enzymes, significantly decreased $\left(\log _{2} \mathrm{FC}=\right.$ -0.74 , adj. $P=0.007 ; \log _{2} \mathrm{FC}=-1.03$, adj. $P=0.026$, respectively). This indicated that the normal function of oxidation-reduction was compromised. Gadd45a and Gadd45b, which are growth arrest and DNA damage response genes, also increased $\left(\log _{2} \mathrm{FC}=1.13\right.$, adj. $P=0.045 ; \log _{2} \mathrm{FC}=1.29$, adj. $P=0.024$, respectively). This indicated that kidney cells responded to cisplatin-induced DNA damage by cell cycle arrest and initiated the DNA repair system, in which case, the p53 signaling pathway. Tnfrsf $12 a$ and Tnfrsf $10 b$ (also known as Dr5), which belong to tumor necrosis factor receptor superfamily and transduces apoptosis signals, significantly increased in CP-AKI group $\left(\log _{2} \mathrm{FC}=1.50\right.$, adj. $P=0.003 ; \log _{2} \mathrm{FC}=1.45$, adj. $P=0.006$, respectively). Caspase 8 also significantly increased in CP-AKI group $\left(\log _{2} \mathrm{FC}=0.74\right.$, adj. $P=0.044)$. This indicated that cisplatin-induced intrinsic apoptosis through Caspase 8 by upregulating Tnfrsf $10 \mathrm{~b}$ in the CP-AKI group. The results demonstrated that the apoptosis process was initiated in the CP-AKI group after $24 \mathrm{~h}$ of being treated with cisplatin. Bcl10 contains a caspase recruitment domain (CARD) and has been shown to induce apoptosis and activate NF- $\mathrm{B}$. Bcl10 slightly increased in CP-AKI group ( $\log _{2} \mathrm{FC}=0.70$, adj. $P=0.159$ ).

However, this process is reversible if cell oxidative stress can be alleviated. In PBS and PSCZ pre-treated group, Bax and Bak1 showed the tendency to return to normal level $\left(\right.$ Bax: $\log _{2} \mathrm{FC}=$ 0.89 , adj. $P=0.260$; $\log _{2} \mathrm{FC}=0.52$, adj. $P=0.774$, respectively; $B a k 1: \log _{2} \mathrm{FC}=0.39$, adj. $P=0.836 ; \log _{2} \mathrm{FC}=0.11$, adj. $P=1.000$, respectively), Tnfrsf $12 a$ and Tnfrsf $10 b$ also showed the tendency to return to normal level (Tnfrsf12a: $\log _{2} \mathrm{FC}=0.54$, adj. $P=0.500$; $\log _{2} \mathrm{FC}=0.13$, adj. $P=1.000$, respectively; Tnfrsf $10 b$ : $\log _{2} \mathrm{FC}=$ 0.99 , adj. $P=0.177 ; \log _{2} \mathrm{FC}=0.42$, adj. $P=0.860$, respectively) compared with CP-AKI group. This indicated that renal cell oxidative stress was significantly ameliorated, and cell apoptosis was repressed. Gadd45a and Gadd45b also showed the tendency to return to normal level in PBS and PSCZ pre-treated AKI mouse models (Gadd45a: $\log _{2} \mathrm{FC}=0.35$, adj. $P=0.897 ; \log _{2} \mathrm{FC}=-0.04$, adj. $P=1.000$, respectively; Gadd45b: $\log _{2} \mathrm{FC}=0.38$, adj. $P=$ $0.862 ; \log _{2} \mathrm{FC}=0.33$, adj. $P=0.957$, respectively). This indicated cell cycle arrest was also alleviated in both groups. Tadagavadi et al. also reported that PBS had protective effects in AKI models (Tadagavadi and Reeves, 2010). This is consistent with the findings in current study. In the current study, PSCZ showed superior protective effect than PBS.

\section{MATERIALS AND METHODS}

\subsection{Materials}

$\mathrm{Zn}\left(\mathrm{NO}_{3}\right)_{2} \bullet 6\left(\mathrm{H}_{2} \mathrm{O}\right)$, 2-methyl imidazole (2-HmeIM), polyvinylpyrrolidone (PVP), SOD, CAT, and Fluorescein isothiocyanate (FITC) were purchased from Sigma-Aldrich and used as received. Methoxy-PEG-acid $\left(\mathrm{MPEG}_{5000}-\mathrm{COOH}\right)$ and $\mathrm{NH}_{2}-\mathrm{PEG}_{2000}-\mathrm{COOH}$ were purchased from Ponsure Biological. Phosphate buffer solution (PBS) was purchased from Thermo-Fisher (Waltham, MA, United States). LysoTracker Green and Reactive Oxygen Species Assay Kit were purchased from Beyotime (China). All the aqueous solutions were prepared using purified deionized (DI) water purified with a purification system (Direct-Q3, Millipore, United States). The other solvents used in this work were purchased from Sinopharm Chemical Reagent (China) and Aladdin-Reagent (China).

\subsection{Construction of SOD@CAT@ZIF-8 Through Biomimetic Mineralization}

Typically, $4 \mathrm{ml}$ aqueous solution containing HMeIM (990 mg, $12 \mathrm{mM})$ was mixed with $4 \mathrm{ml}$ of $\mathrm{Zn}\left(\mathrm{NO}_{3}\right)_{2} \bullet 6\left(\mathrm{H}_{2} \mathrm{O}\right)$ solution $(52 \mathrm{mg}, 0.175 \mathrm{mM}), 400 \mu \mathrm{l}$ of SOD $(10 \mathrm{mg})$ and CAT $(10 \mathrm{mg})$ solution. The mixture was incubated at room temperature for $5 \mathrm{~min}$, and then the centrifuged pelletes were collected and washed with deionized water. The white solids were subsequently dried at room temperature to obtain the composite SOD@CAT@ZIF-8. The SOD and CAT loading in ZIF-8 were calculated using the following formula:

$$
\text { Protein loading }(\%)=[(\mathrm{A}-\mathrm{B}) / \mathrm{A}] \times 100 \%
$$

$A$ and $B$ represented the amount of protein in the initial solution and washing solution, measured based on the BCA assay.

\subsection{Synthesis of PSCZ}

$\mathrm{MPEG}_{2000}-\mathrm{COOH}$ was connected to the surface of SOD@CAT@ ZIF-8 through a coordination bond between $-\mathrm{COOH}$ group and the $\mathrm{Zn}$ ion to prepare PMs. Briefly, pre-formed CAT@SOD@ZIF8 was dispersed in an $\mathrm{MPEG}_{2000}-\mathrm{COOH}$ solution of $10 \mathrm{mg} / \mathrm{ml}$ using deionized water as the solvent. The mixture was sonicated for $10 \mathrm{~min}$ and stirred at room temperature for $48 \mathrm{~h}$ to form a PSCZ suspension. The white product was collected by centrifugation, followed by washing three times with deionized water and drying at $35^{\circ} \mathrm{C}$ under vacuum.

\subsection{Superoxide Anion Scavenging With PSCZ}

The superoxide anion scavenging activity was assessed with a SOD assay kit (Solarbio, China). The MPEG $2000-S O D @ C A T @$ ZIF-8 (PSCZ), MEPG $2000^{-S O D @ Z I F-8 ~(P S Z), ~ M P E G ~} 2000^{-}$ CAT@ZIF-8 (PCZ) concentrations $(100 \mu \mathrm{g} / \mathrm{ml})$ test was performed according to the instructions provided with the kit. The absorbance at $560 \mathrm{~nm}$ was measured using a quartz colorimetric dish reader after incubating at $37^{\circ} \mathrm{C}$ for $30 \mathrm{~min}$. 


\subsection{PSCZ Scavenger Hydrogen Peroxide}

The superoxide anion scavenging activity was assessed with a CAT assay kit (Solarbio, China). The PSCZ, PSZ, PCZ concentrations $(100 \mu \mathrm{g} / \mathrm{ml})$ test was performed according to the instructions provided with the kit. The absorbance at $405 \mathrm{~nm}$ was measured using a quartz colorimetric dish reader after incubating at room temperature for $10 \mathrm{~min}$.

\subsection{Viability of Cells Protected by PSCZ From Oxidative Stress}

Human embryonic kidney 293 (HEK293) was cultured in Dulbecco's Modified Eagle Medium (DMEM) (1\% penicillin/ streptomycin and $10 \%$ fetal bovine serum) at $37^{\circ} \mathrm{C}$ under $5 \% \mathrm{CO}_{2}$. Using an RTCA E-Plate, HEK293 cells were seeded at a concentration of $1 \times 10^{4}$ cells per well and incubated for $24 \mathrm{~h}$. Next, PSCZ, PSZ, and PCZ dispersed in the cell culture medium at various concentrations $(5,10,20 \mu \mathrm{g} / \mathrm{ml})$ were added to the wells and incubated for $1 \mathrm{~h} \mathrm{H}_{2} \mathrm{O}_{2}$ was then added to the cells to a final concentration of $200 \mu \mathrm{m}$ and incubated for $24 \mathrm{~h}$. Cells with no treatment and treated with only $\mathrm{H}_{2} \mathrm{O}_{2}$ were used as controls compared with only PSCZ, PSZ, and PCZ. The standard RTCA analysis is used to measure cell viability.

\subsection{PSCZ Scavenging of Reactive Oxygen Species in vitro}

$2^{\prime}, 7^{\prime}$-Dichlorofluorescin diacetate (DCFH-DA, D6883, SigmaAldrich, United States), an oxidation-sensitive fluorescent dye, was used to detect the intracellular ROS level, according to the literature. Briefly, DCFH-DA is a non-fluorescent chemical compound that could freely diffuse through the cell membrane and be hydrolyzed by intracellular esterase to DCFH. The intracellular ROS could oxidize the non-fluorescent DCFH to fluorescent DCF. Therefore, the quantity of intracellular ROS is correlated with the fluorescent intensity of DCF. After incubation with $\mathrm{H}_{2} \mathrm{O}_{2}$ for $24 \mathrm{~h}$, cells were gently rinsed thrice with a serumfree medium to remove the free PSCZ. Then, a final concentration of $10 \mu \mathrm{M}$ of DCFH-DA in serum-free medium was added to the cells and incubated in the dark at $37^{\circ} \mathrm{C}$ for $30 \mathrm{~min}$. Afterward, the cells were washed with serum-free medium thrice to remove the unloaded DCFH-DA probe, then were scanned using a laser confocal microscope (Zeiss LSM780, Germany). Flow cytometry analysis was used to quantify the intracellular ROS levels.

Next, cells seeded in six-well plates were stained with Annexin V-FITC apoptosis detection kit (A211-01, Vazyme, China) to detect the ratio of apoptotic and necrotic cells. Briefly, HEK293 cells in a well were collected, washed with cold PBS, and resuspended in $195 \mu \mathrm{l}$ binding buffer after incubation with $\mathrm{H}_{2} \mathrm{O}_{2}$ for $24 \mathrm{~h}$. Then, $5 \mu \mathrm{l}$ Annexin V-FITC and $10 \mu \mathrm{l}$ PI were sequentially added to the cell suspension and incubated at room temperature in the dark for $15 \mathrm{~min}$. After that, cells were analyzed by the Flow cytometer (NovoCyte, Agilent, United States). At least 50,000 cells were analyzed in each sample.

Finally, we used the mitochondrial membrane potential detection kit JC-1 (C2006, Beyotime, China) probe method to detect mitochondrial membrane potential changes. HEK293 cells were seeded separately into confocal laser Petri dishes. Follow the instructions provided with the kit. After incubation with $\mathrm{H}_{2} \mathrm{O}_{2}$ for $24 \mathrm{~h}$, cells were gently rinsed thrice with PBS to remove the free PSCZ. Then add $1 \mathrm{ml}$ of JC- 1 dye solution and mix well. Then the cells were incubated in an incubator at $37^{\circ} \mathrm{C}$ for $20 \mathrm{~min}$. An appropriate amount of JC-1 staining buffer $(1 \times)$ was prepared by adding $1 \mathrm{ml}$ of JC- 1 staining buffer $(5 \times)$ to $4 \mathrm{ml}$ of distilled water and placed in an ice bath during incubation. At the end of incubation at $37^{\circ} \mathrm{C}$, the supernatant was aspirated, washed twice with JC- 1 staining buffer $(1 \times)$, and then $2 \mathrm{ml}$ cell culture solution was added, which could contain serum and phenol red. Finally, using a laser confocal microscope (Zeiss LSM780, Germany).

\subsection{PSCZ Scavenging of Reactive Oxygen Species in vitro}

\subsubsection{Cisplatin-Induced Acute Kidney Injury Model}

Female BALB/c mice aged 6-8 weeks were selected, all mice were received intraperitoneal injections of cisplatin $(20 \mathrm{mg} / \mathrm{kg})$. Twenty four hours after injections, the mice in each group were sacrificed to monitor the model development. The blood samples and renal tissues were obtained and analyzed. The mice were intravenously injected with PSCZ ( $15 \mathrm{mg} / \mathrm{kg}$ in $100 \mu \mathrm{l} \mathrm{PBS})$ at $1 \mathrm{~h}$ after intraperitoneal injections of cisplatin for the treatment group.

\subsubsection{Treatment of Cisplatin-Induced-Acute Kidney Injury Mice}

One hour after the AKI model induction, different treatments were performed on AKI model mice: group 1 was healthy mice $(n=5)$; group 2 was AKI mice (PBS, $n=5$ ); group 3 was AKI mice treated with $1 \times$ PBS $(n=5)$; group 4 was AKI mice treated with PSCZ $(15 \mathrm{mg} / \mathrm{kg}$ in $100 \mu \mathrm{l} \mathrm{PBS}, n=5)$. After $24 \mathrm{~h}$ p.i, blood samples and tissues of major organs were collected, the treatment group was compared with healthy mice to evaluate kidney function.

\subsubsection{Kidney Function Test}

After euthanizing the mice, blood samples were collected in lithium heparin tubes. The blood serum was collected after centrifuging the blood samples at $2,000 \mathrm{~g}$ for $15 \mathrm{~min}$ at $4^{\circ} \mathrm{C}$. Finally, the samples were sent to the Laboratory Department of the First Affiliated Hospital of Zhengzhou University for analysis of blood urea nitrogen (BUN) levels and blood creatinine (CRE) levels.

\subsubsection{Hematoxylin and Eosin Staining of Kidney Sections}

In the AKI model, mice were euthanized to harvest the kidneys at the time-point of interest. Next, the kidneys were fixed with paraformaldehyde (4\% in PBS) and embedded in paraffin wax, sliced, and stained with H\&E stain in the Laboratory Animal Center of Zhengzhou University.

3.8.5 Analysis of the Therapeutic Effect in Renal Tissue Kidneys from each group were snap-frozen and stored at $-80^{\circ} \mathrm{C}$ until use. Kidney homogenates were prepared according to the 
protocols of different assays. SOD level and CAT level were assessed with a SOD assay kit and CAT assay kit (SigmaAldrich, United States). Kidney tissues of mice in each group were sliced using a frozen micro-slicer to evaluate ROS's scavenging effect. Frozen kidney tissue slides (about $5 \mu \mathrm{m}$ thickness) were washed with PBS. One of the slides was stained with $1 \mathrm{mM}$ DCFH-DA for $30 \mathrm{~min}$ to detect superoxide formation, and another slide was stained with TNFa- and IL1 $\beta$ antibodies and DAPI to evaluate the development of inflammation in the tissues. Then a cover glass was applied to each slide using Vectashield mounting medium. Finally, a laser confocal microscope was used to observe the results.

\subsubsection{RNA Sequencing}

Mice were sacrificed to harvest the kidneys. The kidneys were washed thrice with saline and snap-frozen in liquid nitrogen. Total RNA was extracted by using the TIANGEN Animal Tissue Total RNA Extraction Kit (DP419, TIANGEN, China). RNA integrity was verified by Agilent 4200 Bioanalyzer and quantified using ND-2000 (Nanodrop Technology). Cell rRNA was removed by using MGIEasy rRNA removal kit. Library construction was carried out using the MGIEasy RNA Library Prep Set (96 RXN) according to the manufacturer's protocol. Libraries were visualized on the Agilent 4200 Bioanalyzer to check insert size and quantified by using the Qubit Fluorometers to determine the concentration. Libraries were pooled and loaded on the flow cell to run on MGISEQ-2000 sequencer as paired-end read for 150 cycles on each side.

\subsection{Bioinformatics Analysis}

\subsubsection{Transcriptome Sequencing and Mapping}

Clean reads were mapped to Mus musculus reference genome (GRCm38) with STAR aligner (version 2.6.1d) (Dobin et al., 2013). Transcript-level gene expression was estimated by Salmon (version 0.14.1) (Patro et al., 2017). Transcript per million (TPM) values were calculated using tximport. A community-developed pipeline called bcbio-nextgen was used to automate the upstream bioinformatic analysis (Guimera, 2011).

\subsubsection{Identification and Functional Annotation of Differentially Expressed Genes}

The R package bcbioRNASeq can take the bcbio-nextgen output as input and generate count matrices, identify DEGs, conduct functional annotations and visualize the results (Steinbaugh et al., 2018). DEGs were identified for each group compared with the control group using DESeq2, (Love et al., 2014) with a fold-change threshold of 1 and $P$ values cutoff of 0.05 . Functional annotation of DEGs was performed using the clusterProfiler $\mathrm{R}$ package to identify associated pathways in AKI (Yu et al., 2012). We further explored the mechanism of $\mathrm{AKI}$ and whether PSCZ can reverse the unfavorable changes in transcriptional level.

\subsection{Characterization}

T Fourier transform infrared spectra (FT-IR) were measured on a Shimadzu FTIR 8400S spectrometer. Scanning electron microscopy (SEM) images were captured on a Hitachi FESEM S-4800 instrument with an acceleration voltage of $3 \mathrm{kV}$. The samples were prepared by depositing sample dispersion onto a freshly cleaved silicon wafers surface. Powder X-ray diffraction (PXRD) patterns were collected on a PANalytical B.V. Empyrean powder diffractometer, in which data were collected from $5^{\circ}$ to $45^{\circ}$ at a scan rate of $15^{\circ} / \mathrm{min}$.

\section{DATA AVAILABILITY STATEMENT}

The datasets presented in this study can be found in online repositories. RNA-seq sequencing results can be found in the NODE database using accession number OEP000285.

\section{ETHICS STATEMENT}

The animal study was reviewed and approved by the Experimental Animal Center of Zhengzhou University.

\section{AUTHOR CONTRIBUTIONS}

WS, JG, TH, and WJ conceived the idea, provided funding support, interpreted the results, and critically revised and finalized the manuscript. $\mathrm{XH}$ : designed and performed experiments, organized the data, and wrote the manuscript. JS: analyzed transcriptome sequencing data and interpreted results wrote and revised the manuscript. JZ: constructed the mouse model and performed the experiments. ZW: provided materials and helped to construct the mouse model. SZ: conducted molecular biological experiments. RL: performed data analysis, generated figures, and organized data.

\section{FUNDING}

We appreciate the financial support from the National Natural Science Foundation of China (No. 81901882), China postdoctoral science foundation (2019M663062), Henan Province Medical Science and Technology Public Relations Plan Province Department joint construction project (SBGJ2018041, LHGJ20190329), and Education Department of Henan Province (20A430026), the Collaborative Innovation Project of Zhengzhou (Zhengzhou University) (No. 20XTZX05014). Data analysis was supported by the Supercomputing Center in Zhengzhou University (Zhengzhou).

\section{SUPPLEMENTARY MATERIAL}

The Supplementary Material for this article can be found online at: https:/www.frontiersin.org/articles/10.3389/fbioe.2021.800428/ full\#supplementary-material 


\section{REFERENCES}

Arany, I., and Safirstein, R. L. (2003). Cisplatin Nephrotoxicity. Semin. Nephrol. 23 (5), 460-464. doi:10.1016/s0270-9295(03)00089-5

Bellomo, R., Kellum, J. A., and Ronco, C. (2012). Acute Kidney Injury. Lancet 380 (9843), 756-766. doi:10.1016/s0140-6736(11)61454-2

Bolisetty, S., Traylor, A., Joseph, R., Zarjou, A., and Agarwal, A. (2016). Proximal Tubule-Targeted Heme Oxygenase-1 in Cisplatin-Induced Acute Kidney Injury. Am. J. Physiol. Renal Physiol. 310 (5), F385-F394. doi:10.1152/ ajprenal.00335.2015

Cetin, I., and Topcul, M. (2019). Evaluation of the Cytotoxic Effect of Ly2109761 on HeLa Cells Using the xCELLigence RTCA System. Oncol. Lett. 17 (1), 683-687. doi:10.3892/ol.2018.9556

Chen, D., Suo, M., Guo, J., Tang, W., Jiang, W., Liu, Y., et al. (2021). Development of MOF “Armor-Plated" Phycocyanin and Synergistic Inhibition of Cellular Respiration for Hypoxic Photodynamic Therapy in Patient-Derived Xenograft Models. Adv. Healthc. Mater. 10 (3), 2001577. doi:10.1002/adhm.202001577

Dasari, S., and Bernard Tchounwou, P. (2014). Cisplatin in Cancer Therapy: Molecular Mechanisms of Action. Eur. J. Pharmacol. 740, 364-378. doi:10.1016/j.ejphar.2014.07.025

Dennis, J., and Witting, P. (2017). Protective Role for Antioxidants in Acute Kidney Disease. Nutrients 9 (7), 718. doi:10.3390/nu9070718

Dobin, A., Davis, C. A., Schlesinger, F., Drenkow, J., Zaleski, C., Jha, S., et al. (2013). STAR: Ultrafast Universal RNA-Seq Aligner. Bioinformatics 29 (1), 15-21. doi:10.1093/bioinformatics/bts635

Du, B., Jiang, X., Das, A., Zhou, Q., Yu, M., Jin, R., et al. (2017). Glomerular Barrier Behaves as an Atomically Precise Bandpass Filter in a Sub-Nanometre Regime. Nat. Nanotech 12 (11), 1096-1102. doi:10.1038/nnano.2017.170

Feng, D., Liu, T.-F., Su, J., Bosch, M., Wei, Z., Wan, W., et al. (2015). Stable MetalOrganic Frameworks Containing Single-Molecule Traps for Enzyme Encapsulation. Nat. Commun. 6, 5979. doi:10.1038/ncomms6979

Ferreira, C. A., Ni, D., Rosenkrans, Z. T., and Cai, W. (2018). Scavenging of Reactive Oxygen and Nitrogen Species with Nanomaterials. Nano Res. 11 (10), 4955-4984. doi:10.1007/s12274-018-2092-y

Guimera, R. V. (2011). Bcbio-Nextgen: Automated, Distributed Next-Gen Sequencing Pipeline. EMBnet J. 17 (B), 30.

Hao, C., Qu, A., Xu, L., Sun, M., Zhang, H., Xu, C., et al. (2019). Chiral MoleculeMediated Porous CuxO Nanoparticle Clusters with Antioxidation Activity for Ameliorating Parkinson's Disease. J. Am. Chem. Soc. 141 (2), 1091-1099. doi:10.1021/jacs.8b11856

Huang, S., Kou, X., Shen, J., Chen, G., and Ouyang, G. (2020). “Armor-Plating” Enzymes with Metal-Organic Frameworks (MOFs). Angew. Chem. Int. Ed. 59 (23), 8786-8798. doi:10.1002/anie.201916474

Ighodaro, O. M., and Akinloye, O. A. (2018). First Line Defence Antioxidants-Superoxide Dismutase (SOD), Catalase (CAT) and Glutathione Peroxidase (GPX): Their Fundamental Role in the Entire Antioxidant Defence Grid. Alexandria J. Med. 54 (4), 287-293. doi:10.1016/j.ajme.2017.09.001

Jiang, W., Wang, X., Chen, J., Liu, Y., Han, H., Ding, Y., et al. (2017). Deuterohemin-Peptide Enzyme Mimic-Embedded Metal-Organic Frameworks through Biomimetic Mineralization with Efficient ATRP Catalytic Activity. ACS Appl. Mater. Inter. 9 (32), 26948-26957. doi:10.1021/acsami.7b09218

Liang, K., Ricco, R., Doherty, C. M., Styles, M. J., Bell, S., Kirby, N., et al. (2015). Biomimetic Mineralization of Metal-Organic Frameworks as Protective Coatings for Biomacromolecules. Nat. Commun. 6, 7240. doi:10.1038/ ncomms 8240

Liang, W., Wied, P., Carraro, F., Sumby, C. J., Nidetzky, B., Tsung, C.-K., et al. (2021). Metal-Organic Framework-Based Enzyme Biocomposites. Chem. Rev. 121 (3), 1077-1129. doi:10.1021/acs.chemrev.0c01029

Liu, J., Liang, J., Xue, J., and Liang, K. (2021). Metal-Organic Frameworks as a Versatile Materials Platform for Unlocking New Potentials in Biocatalysis. Small 17 (32), 2100300. doi:10.1002/smll.202100300

Liu, T., Xiao, B., Xiang, F., Tan, J., Chen, Z., Zhang, X., et al. (2020). Ultrasmall Copper-Based Nanoparticles for Reactive Oxygen Species Scavenging and Alleviation of Inflammation Related Diseases. Nat. Commun. 11 (1), 2788. doi:10.1038/s41467-020-16544-7
Love, M. I., Huber, W., and Anders, S. (2014). Moderated Estimation of Fold Change and Dispersion for RNA-Seq Data with DESeq2. Genome Biol. 15 (12), 550-621. doi:10.1186/s13059-014-0550-8

Lu, K., Aung, T., Guo, N., Weichselbaum, R., and Lin, W. (2018). Nanoscale MetalOrganic Frameworks for Therapeutic, Imaging, and Sensing Applications. Adv. Mater. 30 (37), 1707634. doi:10.1002/adma.201707634

Manohar, S., and Leung, N. (2018). Cisplatin Nephrotoxicity: A Review of the Literature. J. Nephrol. 31 (1), 15-25. doi:10.1007/s40620-017-0392-z

Martindale, J. L., and Holbrook, N. J. (2002). Cellular Response to Oxidative Stress: Signaling for Suicide and Survival. J. Cell. Physiol. 192 (1), 1-15. doi:10.1002/ jcp. 10119

Meng, X.-M., Ren, G.-L., Gao, L., Yang, Q., Li, H.-D., Wu, W.-F., et al. (2018). NADPH Oxidase 4 Promotes Cisplatin-Induced Acute Kidney Injury via ROSMediated Programmed Cell Death and Inflammation. Lab. Invest. 98 (1), 63-78. doi:10.1038/labinvest.2017.120

Molitoris, B. A., Levin, A., Levin, A., Warnock, D. G., Joannidis, M., Mehta, R. L., et al. (2007). Improving Outcomes of Acute Kidney Injury: Report of an Initiative. Nat. Rev. Nephrol. 3 (8), 439-442. doi:10.1038/ncpneph0551

Nath, K. A., and Norby, S. M. (2000). Reactive Oxygen Species and Acute Renal Failure. Am. J. Med. 109 (8), 665-678. doi:10.1016/S0002-9343(00) 00612-4

Ni, D., Jiang, D., Kutyreff, C. J., Lai, J., Yan, Y., Barnhart, T. E., et al. (2018). Molybdenum-Based Nanoclusters Act as Antioxidants and Ameliorate Acute Kidney Injury in Mice. Nat. Commun. 9 (1), 5421. doi:10.1038/s41467-01807890-8

Ozkok, A., and Edelstein, C. L. (2014). Pathophysiology of Cisplatin-Induced Acute Kidney Injury. Biomed. Res. Int. 2014, 1-17. doi:10.1155/2014/967826

Pabla, N., and Dong, Z. (2008). Cisplatin Nephrotoxicity: Mechanisms and Renoprotective Strategies. Kidney Int. 73 (9), 994-1007. doi:10.1038/ sj.ki.5002786

Paller, M. S., Hoidal, J. R., and Ferris, T. F. (1984). Oxygen Free Radicals in Ischemic Acute Renal Failure in the Rat. J. Clin. Invest. 74 (4), 1156-1164. doi:10.1172/JCI111524

Patro, R., Duggal, G., Love, M. I., Irizarry, R. A., and Kingsford, C. (2017). Salmon Provides Fast and Bias-Aware Quantification of Transcript Expression. Nat. Methods 14 (4), 417-419. doi:10.1038/nmeth.4197

Pavlakou, P., Liakopoulos, V., Eleftheriadis, T., Mitsis, M., and Dounousi, E. (2017). Oxidative Stress and Acute Kidney Injury in Critical Illness: Pathophysiologic Mechanisms-Biomarkers-Interventions, and Future Perspectives. Oxid. Med. Cell Longev. 2017, 1-11. doi:10.1155/2017/6193694

Ratliff, B. B., Abdulmahdi, W., Pawar, R., and Wolin, M. S. (2016). Oxidant Mechanisms in Renal Injury and Disease. Antioxid. Redox Signal. 25 (3), 119-146. doi:10.1089/ars.2016.6665

Rewa, O., and Bagshaw, S. M. (2014). Acute Kidney Injury-Epidemiology, Outcomes and Economics. Nat. Rev. Nephrol. 10 (4), 193-207. doi:10.1038/ nrneph.2013.282

Rosenkrans, Z. T., Sun, T., Jiang, D., Chen, W., Barnhart, T. E., Zhang, Z., et al. (2020). Selenium-Doped Carbon Quantum Dots Act as Broad-Spectrum Antioxidants for Acute Kidney Injury Management. Adv. Sci. 7 (12), 2000420. doi:10.1002/advs.202000420

Sastre, J., Pallardó, F. V., García de la Asunción, J., and Viña, J. (2000). Mitochondria, Oxidative Stress and Aging. Free Radic. Res. 32 (3), 189-198. doi:10.1080/10715760000300201

Savelieff, M. G., Nam, G., Kang, J., Lee, H. J., Lee, M., and Lim, M. H. (2019). Development of Multifunctional Molecules as Potential Therapeutic Candidates for Alzheimer's Disease, Parkinson's Disease, and Amyotrophic Lateral Sclerosis in the Last Decade. Chem. Rev. 119 (2), 1221-1322. doi:10.1021/acs.chemrev.8b00138

Schieber, M., and Chandel, N. S. (2014). ROS Function in Redox Signaling and Oxidative Stress. Curr. Biol. 24 (10), R453-R462. doi:10.1016/j.cub.2014.03.034

Sharp, C. N., Doll, M. A., Dupre, T. V., Shah, P. P., Subathra, M., Siow, D., et al. (2016). Repeated Administration of Low-Dose Cisplatin in Mice Induces Fibrosis. Am. J. Physiol. Renal Physiol. 310 (6), F560-F568. doi:10.1152/ajprenal.00512.2015

Sharp, C. N., and Siskind, L. J. (2017). Developing Better Mouse Models to Study Cisplatin-Induced Kidney Injury. Am. J. Physiol. Renal Physiol. 313 (4), F835-F841. doi:10.1152/ajprenal.00285.2017

Shieh, F.-K., Wang, S.-C., Yen, C.-I., Wu, C.-C., Dutta, S., Chou, L.-Y., et al. (2015). Imparting Functionality to Biocatalysts via Embedding Enzymes into 
Nanoporous Materials by a de novo Approach: Size-Selective Sheltering of Catalase in Metal-Organic Framework Microcrystals. J. Am. Chem. Soc. 137 (13), 4276-4279. doi:10.1021/ja513058h

Simon-Yarza, T., Giménez-Marqués, M., Mrimi, R., Mielcarek, A., Gref, R., Horcajada, P., et al. (2017). A Smart Metal-Organic Framework Nanomaterial for Lung Targeting. Angew. Chem. Int. Ed. 56 (49), 15565-15569. doi:10.1002/anie.201707346

Steinbaugh, M. J., Pantano, L., Kirchner, R. D., Barrera, V., Chapman, B. A., Piper, M. E., et al. (2018). bcbioRNASeq: R Package for Bcbio RNA-Seq Analysis. F1000Res 6 (1976), 1976. doi:10.12688/f1000research.12093.2

Sun, C.-Y., Qin, C., Wang, X.-L., Yang, G.-S., Shao, K.-Z., Lan, Y.-Q., et al. (2012). Zeolitic Imidazolate Framework-8 as Efficient pH-Sensitive Drug Delivery Vehicle. Dalton Trans. 41 (23), 6906-6909. doi:10.1039/c2dt30357d

Sun, H., Zhou, Y., Ren, J., and Qu, X. (2018). Carbon Nanozymes: Enzymatic Properties, Catalytic Mechanism, and Applications. Angew. Chem. Int. Ed. 57 (30), 9224-9237. doi:10.1002/anie.201712469

Suresh, K., and Matzger, A. J. (2019). Enhanced Drug Delivery by Dissolution of Amorphous Drug Encapsulated in a Water Unstable Metal-Organic Framework (MOF). Angew. Chem. Int. Ed. 58 (47), 16790-16794. doi:10.1002/anie.201907652

Sureshbabu, A., Ryter, S. W., and Choi, M. E. (2015). Oxidative Stress and Autophagy: Crucial Modulators of Kidney Injury. Redox Biol. 4, 208-214. doi:10.1016/j.redox.2015.01.001

Susantitaphong, P., Cruz, D. N., Cerda, J., Abulfaraj, M., Alqahtani, F., Koulouridis, I., et al. (2013). World Incidence of AKI: A Meta-Analysis. Clin. J. Am. Soc. Nephrol. 8 (9), 1482-1493. doi:10.2215/cjn.00710113

Tadagavadi, R. K., and Reeves, W. B. (2010). Renal Dendritic Cells Ameliorate Nephrotoxic Acute Kidney Injury. Jasn 21 (1), 53-63. doi:10.1681/asn.2009040407

Tripathy, S. P., Subudhi, S., and Parida, K. (2021). Inter-MOF Hybrid (IMOFH): A Concise Analysis on Emerging Core-Shell Based Hierarchical and Multifunctional Nanoporous Materials. Coord. Chem. Rev. 434, 213786. doi:10.1016/j.ccr.2021.213786

Velásquez-Hernández, M. d. J., Linares-Moreau, M., Astria, E., Carraro, F., Alyami, M. Z., Khashab, N. M., et al. (2021). Towards Applications of Bioentities@MOFs in Biomedicine. Coord. Chem. Rev. 429, 213651. doi:10.1016/j.ccr.2020.213651

Wang, H., Wan, K., and Shi, X. (2019). Recent Advances in Nanozyme Research. Adv. Mater. 31 (45), 1805368. doi:10.1002/adma.201805368

Wang, Q., Zhang, X., Huang, L., Zhang, Z., and Dong, S. (2017). GOx@ZIF8(NiPd) Nanoflower: An Artificial Enzyme System for Tandem Catalysis. Angew. Chem. Int. Ed. 56 (50), 16082-16085. doi:10.1002/anie.201710418

Wu, J., Wang, X., Wang, Q., Lou, Z., Li, S., Zhu, Y., et al. (2019). Nanomaterials with Enzyme-like Characteristics (Nanozymes): Next-Generation Artificial Enzymes (II). Chem. Soc. Rev. 48 (4), 1004-1076. doi:10.1039/C8CS00457A

$\mathrm{Xu}, \mathrm{W}$., Jiao, L., Wu, Y., Hu, L., Gu, W., and Zhu, C. (2021). Metal-Organic Frameworks Enhance Biomimetic Cascade Catalysis for Biosensing. Adv. Mater. 33 (22), 2005172. doi:10.1002/adma.202005172
Xu, Y., Fei, J., Li, G., Yuan, T., Xu, X., and Li, J. (2019). Nanozyme-Catalyzed Cascade Reactions for Mitochondria-Mimicking Oxidative Phosphorylation. Angew. Chem. Int. Ed. 58 (17), 5572-5576. doi:10.1002/anie.201813771

Yah, C. S., and Simate, G. S. (2020). Engineered Nanoparticle Bio-Conjugates Toxicity Screening: The xCELLigence Cells Viability Impact. Bioimpacts 10 (3), 195-203. doi:10.34172/bi.2020.24

Yasui, H., and Sakurai, H. (2000). Chemiluminescent Detection and Imaging of Reactive Oxygen Species in Live Mouse Skin Exposed to UVA. Biochem. Biophys. Res. Commun. 269 (1), 131-136. doi:10.1006/bbrc.2000.2254

Yu, B., Wei, H., He, Q., Ferreira, C. A., Kutyreff, C. J., Ni, D., et al. (2018). Efficient Uptake of 177 Lu-Porphyrin-PEG Nanocomplexes by Tumor Mitochondria for Multimodal-Imaging-Guided Combination Therapy. Angew. Chem. Int. Ed. 57 (1), 218-222. doi:10.1002/anie.201710232

Yu, G., Wang, L.-G., Han, Y., and He, Q.-Y. (2012). clusterProfiler: An R Package for Comparing Biological Themes Among Gene Clusters. Omics: a J. Integr. Biol. 16 (5), 284-287. doi:10.1089/omi.2011.0118

Yu, H., Jin, F., Liu, D., Shu, G., Wang, X., Qi, J., et al. (2020). ROS-Responsive Nano-Drug Delivery System Combining Mitochondria-Targeting Ceria Nanoparticles with Atorvastatin for Acute Kidney Injury. Theranostics 10 (5), 2342-2357. doi:10.7150/thno.40395

Zhuang, J., Duan, Y., Zhang, Q., Gao, W., Li, S., Fang, R. H., et al. (2020). Multimodal Enzyme Delivery and Therapy Enabled by Cell Membrane-Coated Metal-Organic Framework Nanoparticles. Nano Lett. 20 (5), 4051-4058. doi:10.1021/acs.nanolett.0c01654

Zhuang, J., Gong, H., Zhou, J., Zhang, Q., Gao, W., Fang, R. H., et al. (2020). Targeted Gene Silencing In Vivo by Platelet Membrane-Coated MetalOrganic Framework Nanoparticles. Sci. Adv. 6 (13), eaaz6108. doi:10.1126/sciadv.aaz6108

Conflict of Interest: The authors declare that the research was conducted in the absence of any commercial or financial relationships that could be construed as a potential conflict of interest.

Publisher's Note: All claims expressed in this article are solely those of the authors and do not necessarily represent those of their affiliated organizations, or those of the publisher, the editors and the reviewers. Any product that may be evaluated in this article, or claim that may be made by its manufacturer, is not guaranteed or endorsed by the publisher.

Copyright () 2022 Hou, Shi, Zhang, Wang, Zhang, Li, Jiang, Huang, Guo and Shang This is an open-access article distributed under the terms of the Creative Commons Attribution License (CC BY). The use, distribution or reproduction in other forums is permitted, provided the original author(s) and the copyright owner(s) are credited and that the original publication in this journal is cited, in accordance with accepted academic practice. No use, distribution or reproduction is permitted which does not comply with these terms. 